\title{
Evidence of asymmetric beaming in a piecewise-linear propagation channel
}

\author{
T. F. Gundogdu, ${ }^{1, *}$ M. Gokkavas, ${ }^{1}$ A. E. Serebryannikov, ${ }^{2}$ and E. Ozbay ${ }^{1,3}$ \\ ${ }^{1}$ Nanotechnology Research Center (NANOTAM), Bilkent University, 06800 Ankara, Turkey \\ ${ }^{2}$ Division of Physics of Nanostructures, Faculty of Physics, Adam Mickiewicz University, 61-614 Poznań, Poland \\ ${ }^{3}$ Department of Physics, Department of Electrical Engineering, and National Institute of Materials Science and Nanotechnology (UNAM), \\ Bilkent University, 06800 Ankara, Turkey \\ ${ }^{*}$ Corresponding author: tamara@bilkent.edu.tr
}

Received 25 January 2021; revised 11 May 2021; accepted 23 May 2021; posted 24 May 2021 (Doc. ID 420297); published 14 June 2021

\begin{abstract}
Asymmetric beaming in a piecewise-linear propagation channel is demonstrated for a single photonic-crystal prism at Gaussian-beam illumination. The used hybrid refractiondiffraction mechanism exploits oblique incidence, the first-negative-order deflection at the longer interface, and asymmetry in coupling at the exit interfaces and does not need blocking of transmission by dispersion in the backward illumination case. The Floquet-Bloch mode with left-handed behavior and nearly circular equifrequency dispersion contours is utilized. The outgoing waves may have significantly different spatial distributions for the forward and backward illumination cases, yielding asymmetry in the beaming regime. (c) 2021 Optical Society of America
\end{abstract}

https://doi.org/10.1364/OL.420297

The interest in directional selectivity of electromagnetic and acoustic waves has been growing in the last decades. Two groups of approaches have attracted the attention of researchers. The first group is based on asymmetric transmission (AT), a Lorentz reciprocal phenomenon that appears as a result of asymmetry in coupling [1-6]. The second ones are based on nonmagnetic nonreciprocity enabled by spatio-temporal modulation of material parameters [7]. The former and the latter use the different underlying physics, and thus suggest different physical scenarios and operation regimes. In particular, AT devices can yield various scenarios of directional selectivity that are appropriate for diode(like) operation. At the same time, AT does not enable optical isolators, i.e., devices in which blocking transmission in one of the two opposite directions may occur in the case of a single and straight propagation channel. It is noticeable that the ambiguous use of the terminology by certain ones and the nonjustified expectations of the others led in the past to some misunderstandings of the goals and results of AT studies, e.g., see [8]. AT requires breaking of the spatial inversion symmetry. and thus can be obtained in various structures capable of efficient conversion of the incident-wave energy to different diffraction orders [2], polarization states [9], and waveguide modes [10], while the same illumination regimes are used for forward and backward incidence directions that differ by $180^{\circ}$.
For obtaining asymmetry in transmission, it is sufficient to have a dielectric grating with one-sided corrugations. A wideband AT, at which transmission is suppressed for one of the two opposite directions to yield a high forward-to-backward transmission contrast, can be achieved due to the common effect of diffraction and specific dispersion. Such a combination can be obtained in the nonsymmetric gratings based on photonic crystals (PhCs) [2,11-14], metamaterials [1,15-18], and epsilon-near-zero materials [19]. Generally, the capability in AT is pre-determined by the asymmetry in coupling at the incidence and the exit interface. Interestingly, high transmission can be obtained in PhC gratings for the forward and backward direction at two close frequencies, while strong asymmetry in transmission occurs for each of them $[2,13]$. Thin gratings with subwavelength slits or apertures that may support surface waves [3,20-22] should also be mentioned in the context of AT.

In the prism-shaped configurations, various physical scenarios leading to AT are possible [23-27]. In particular, the diodes enabled by a stack of two different $\mathrm{PhC}$ prisms have been suggested $[24,25,28,29]$. These diodes use the blockage of transmission for one of two opposite incidence directions but, in contrast with $\mathrm{PhC}$ gratings, they may work without higher diffraction orders at the external interfaces. While plane-wave excitation has commonly been used in the earlier-stage studies of AT, the features connected with spatial distribution and wavefront manipulation that enable asymmetry in functionality become crucial at the current stage because of yielding one more degree of freedom. Even a single prism can be capable in AT; see [23]. As demonstrated for beam-type illumination, AT can be achieved even in a solid prism that is a finite-size portion of an isotropic homogeneous dielectric medium. At the same time, prisms based on a dielectric $\mathrm{PhC}$ may serve as low-loss analogs of those made of a homogeneous near-zero-index material [23]. Both $\mathrm{PhC}$ prisms and solid prisms made of a homogeneous material are promising for multichannel circuits with low crosstalk. Noticeably, PhCs remain an excellent platform for new applications [30-32]. In spite of the recent progress in metasurfaces and meta-gratings capable in AT [4,22,33-37], the question of when such quasi-planar structures can and cannot substitute the volume structures remains open. 
In this Letter, the evidence of asymmetric beaming in a single equivalent piecewise-linear propagation channel is demonstrated. We will show that the beam re-shaping (narrowing or widening) occurs at the forward (shorter side) illumination which, together with the specifics of the backward (longer side) illumination, yields strong asymmetry in the beaming regime. It can manifest itself via different spatial distributions of the outgoing beams while transmission remains significant for both the forward and backward illumination cases. For the purposes of numerical demonstration, we use a single prism based on a dielectric-rod $\mathrm{PhC}$ with a rich modal diversity. The prism is designed to have asymmetry in coupling that occurs because the different (sets of) diffraction orders are responsible for the coupling at the front-side and back-side interfaces, and different parts of the tangential wavenumber range contribute to it. As a reminder, in the case of a nonsymmetric PhC grating and plane-wave illumination, AT needs asymmetry in coupling achieved with the help of a higher diffraction order. In the studied case, it is obtained due to the interface location, without additional corrugations. Moreover, beams composing multiple plane-wave components are used. Hence, the conventional coupling analysis based on equifrequency dispersion contours (EFCs) and tangential wavevector conservation cannot fully predict the transmission/scattering scenario, in particular (but not only) because the weights and coupling efficiencies for the individual angular components should be taken into account. Thus, the main attention will be paid to the field distributions. The proposed mechanism uses a Floquet-Bloch (FB) mode with nearly circular EFCs. A higher-order Brillouin zone (BZ) contributes to the coupling at the longer interface. Our mechanism works well at nonzero, but relatively small incidence angles. Transmission through the $\mathrm{PhC}$ prism was simulated by using Lumerical software [38]. Dispersion results were obtained by using a code based on the finite-difference time-domain method.

The introduced refraction-diffraction mechanism is aimed to extend the variety of asymmetric functionality scenarios, a topic currently attracting a lot of interest. It differs from the earlier studies of PhC-prism-based structures with AT features, e.g., in $[24,25]$, in that (1) the transmission is not blocked by dispersion for one of the two used illumination directions, (2) the first negative order creates deflection at the longer interface that dominates zero-order (i.e., refraction) effects, (3) asymmetry assumes that beaming may occur for either both or one of the forward and backward illumination cases, depending on the incidence angle, $\theta$, and (4) single piecewise-linear propagation channel can be introduced as explained below.

Figure 1 shows the general geometry and explains the choice of illumination directions and the idea of a piecewise-linear propagation channel. The $\mathrm{PhC}$ prism was designed by using a heuristic approach and comparing several theoretical performances. A square-lattice $\mathrm{PhC}$ composing dielectric rods of permittivity $\varepsilon=11.4$ (close to $\mathrm{Si}$ ) and a diameter-to-latticeconstant ratio $d / a=0.4$, e.g., $d=400 \mathrm{~nm}$ and $a=1000 \mathrm{~nm}$, has been chosen. The Gaussian beam of waist of $w=10 a$ was used for illumination. TM polarization is considered, i.e., the magnetic field vector is parallel to the rod axes. Geometrical parameters and operating frequency are chosen to enable propagation of the -1 st diffraction order above the interface $\mathrm{B}$. The dominant coupling of one of the FB modes to this order yields a single outgoing (deflected) wave that is a key component of

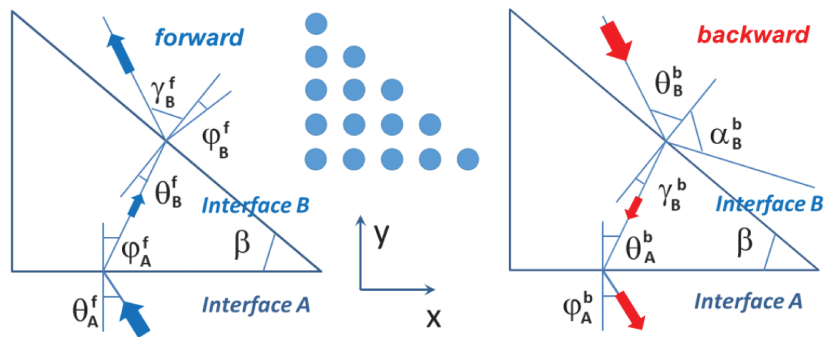

Fig. 1. Schematics showing the general geometry of the $\mathrm{PhC}$ prism and explaining the choice of incidence angles associated with a single piecewise-linear propagation channel.
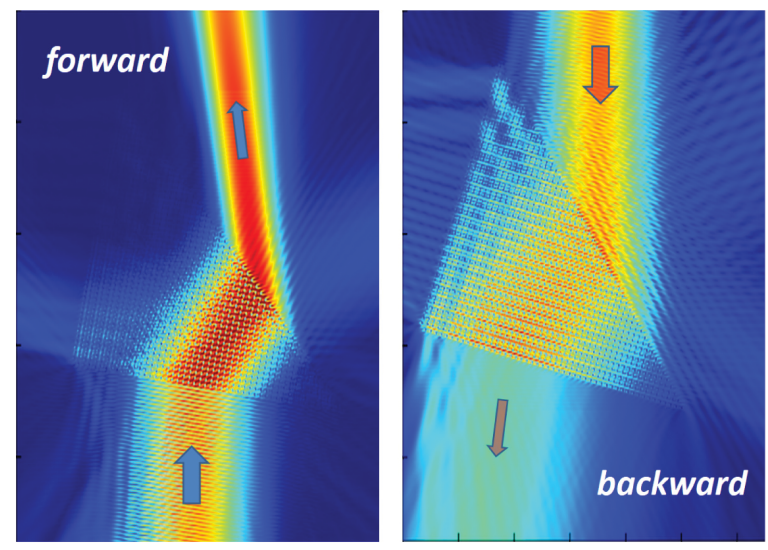

Fig. 2. Map of electric field magnitude at $k a=4.25$ and $\theta=10^{\circ}$ for forward (left panel) and backward (right panel) illumination cases; TM polarization. The prism is rotated here to align incidence direction with a vertical direction; $\beta=45^{\circ}$.

the resulting mechanism of asymmetric beaming. For the sake of definiteness, we call the main propagation path with the best energy localization, as in Fig. 2, single equivalent piecewise-linear propagation channel. If there are multiple propagation paths pre-determined by the Snell law, properties of FB modes of $\mathrm{PhC}$, and diffractions at the interfaces, the main path is assumed to be distinguishable above, below, and inside the prism. The rest of the incident-wave energy may be forwarded to the remaining, i.e., auxiliary propagation paths, or scattered in a nonchanneled manner. Notably, the term channel differs here from its conventional use in optical communications. It is not identical to a diffraction order. Compared to most AT studies in which incidence directions for forward-case and backward-case illuminations differ by $180 \mathrm{deg}$ (for instance, see $[2,5,11,12,25,28]$ ), the illumination direction in the backward case is chosen in this Letter to be opposite to the direction of the outgoing beam of the forward illumination case. This modification is needed to enable a physical situation that is consistent with the concept of the piecewise-linear propagation channel which can be obtained when the specific coupling at the interfaces allows us to suppress or weaken all other formally propagating but unwanted waves. The angle $\theta$ is chosen to make such a channel possible.

Figure 2 presents the map of electric field magnitude, $|E|$, at $\theta=\theta_{A}^{f}=10^{\circ}$. The operating frequency is $f=203 \mathrm{THz}$ $(k a=4.25, k=\omega / c, k$ is the free-space wavenumber, $\omega$ is the angular frequency, and $c$ is the speed of light), is chosen, at which a desired single channel can be obtained. The first negative diffraction order $(m=-1)$ may appear at the interface 
$\mathrm{B}$ and propagate above the prism in this case, but the other orders with $|m| \geq 1$ may not. For the forward-case (here$\Gamma-\mathrm{X}$ interface) illumination, the beam narrowing takes place above the exit (here- $-\Gamma-M$ ) interface. For the backward-case (here-upper-side, $\Gamma-\mathrm{M}$ interface) illumination, the outgoing beam, in contrast, is wider than the incident one. One can see in Fig. 2 that the single piecewise-linear propagation channel can really exist. It is observed that $\theta_{A}^{f} \approx \varphi_{A}^{b}$ and $\theta_{B}^{b} \approx \gamma_{B}^{f}$, but spatial distributions in the exit half-spaces are different. It is important that the demonstrated behavior does not contradict the Lorentz reciprocity. Indeed, the same field distributions at the exit sides in the forward and backward illumination cases would appear when the source in the latter case represents an equivalent source replicating the spatial features of the outgoing wave in the former case. In contrast, we use the beams with identical spatial features in both illumination cases, so that the observed differences in spatial distribution are expectable.

Let us consider coupling at the interfaces $A$ and $B$ in more detail. In the forward illumination case in Fig. 2, $\operatorname{sgn} \theta_{A}^{f} \neq \operatorname{sgn} \varphi_{A}^{f}$ (where $\theta_{A}^{f}$ and $\varphi_{A}^{f}$, respectively, are the incidence and refraction angles at the interface $A$ ) that indicates negative refraction. The period of the incidence interface is $a$ and $|\sin \theta-2 \pi / k a|>1, \theta=\theta_{A}^{f}$. Therefore, diffraction orders with $|m|>0$ may not propagate in the incidence half-space and be coupled to FB modes of the PhC. Since $\left|\theta_{A}^{f}\right|<\left|\varphi_{A}^{f}\right|$, we may conclude that the refraction at the interface $A$ is similar to the one at the interface between air and a medium with the equivalent refraction index $-1<n_{\text {eq }}<0$. As follows from the obtained results, $n_{e q} \approx-0.5$. At the interface $\mathrm{B}$, we observe in Fig. 2 that $\left|\theta_{B}^{f}\right|<\left|\gamma_{B}^{f}\right|\left(\theta_{B}^{f}\right.$ and $\gamma_{B}^{f}$ are the incidence and outgoing wave angles at the interface $B$ ), i.e., it looks like refraction at the interface between a denser and a rarer optical medium. This difference between the interfaces A and B may seem strange at first glance, since the nearly circular EFCs are responsible for coupling.

To clarify the main specifics of coupling for the interfaces A and $\mathrm{B}$, we performed a qualitative analysis by using the dispersion results. Figure 3 presents EFCs for the second FB mode, which is expected to enable the coupling. While an exhaustive dispersion analysis is beyond the scope of this Letter, the shape and location of EFCs, and location of the construction lines clearly indicate the origin of the above-mentioned differences and directional selectivity. The EFCs around $\Gamma$-point are decreasing in size when $k a$ is increased, which indicates left-handedness. This results in a negative sign of refraction, as observed for the interface A in Fig. 2, left panel. In this case, the coupling may be possible at the interface B for two propagating diffraction orders, $m=0$ and $m=-1$, because the interface period is now equal to $\sqrt{2} a$, and higher-order BZs may contribute to the coupling. As follows from the comparison of Figs. 2 and 3, the coupling is strong for $m=-1$, but negligible for $m=0$. Indeed, if coupling to the order $m=0$ would be dominant, a beam should weakly decline from the normal to the interface B (i.e., at $\varphi_{B}^{f}$ instead of $\gamma_{B}^{f}$ ). Note that the directional selectivity with suppressed zero order has been earlier studied in blazed diffraction gratings [39] and $\mathrm{PhC}$ gratings [2].

For the backward-case illumination in Fig. 2, right panel, we have $\theta_{B}^{b}=\gamma_{B}^{f}, \gamma_{B}^{b} \approx \theta_{B}^{f}, \theta_{A}^{b} \approx \varphi_{A}^{f}$, and $\varphi_{A}^{b} \approx \theta_{A}^{f}$. Coupling to the FB mode due to conventional refraction at the interface $\mathrm{B}$ is then not possible because $\left|\sin \theta_{B}^{b} / n_{e q}\right|>1$. Therefore,
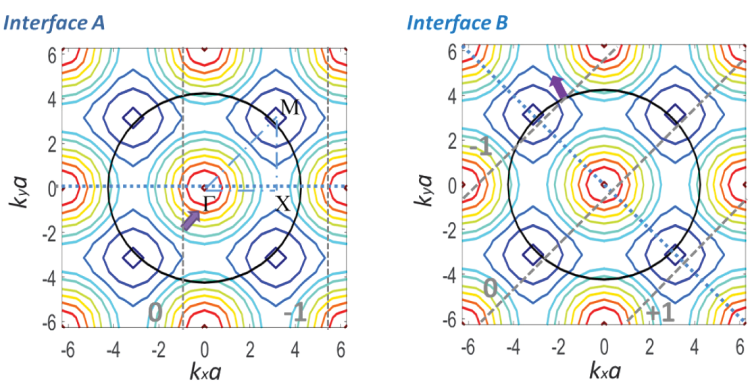

Fig. 3. EFCs for the second FB mode and coupling at interfaces (left panel) A and (right panel) B at forward-case illumination; TM polarization; colors from dark blue to dark red correspond to $k a /(2 \pi)$ varied from 0.6 to 0.78 (step is 0.02 ). The dashed lines and arrows approximately show the construction lines for a selected value of $\theta$ and direction of group velocity, respectively. The circle shows the EFC in air at $k a=4.25$. The coupling needs conservation of the tangential wavenumber. The numbers $-1,0$, and +1 indicate the order, $m$. The dashed-dotted lines show the boundaries of the first BZ. The dotted lines schematically show the orientation of the interfaces.

coupling in this case needs the effect of the orders $|m|>0$ and/or a higher BZ for the order $m=0$, while some of the propagating waves can be suppressed. The beam widening is observed. The angle between the incident and outgoing beams is given by $\delta \approx\left|\theta_{A}^{f}-\gamma_{B}^{f}\right| \approx\left|\theta_{B}^{b}-\varphi_{A}^{b}\right|$. Here we obtain $\delta \approx 7^{\circ}$. Different spatial distributions of the field in the exit half-spaces are expected to occur for the two illumination cases also, because the ranges of variation in the tangential wavenumber of the incident wave, which contribute to the in-coupling and have the same widths, can correspond to the different widths of the ranges of variation in normal and tangential wavenumbers of the wave propagating in the $\mathrm{PhC}$ and to the different effective widths of the angular spectrum of the outgoing wave. A detailed analysis will be done at the next steps to clarify this issue, as well as possible effects of interferences, other $\mathrm{FB}$ modes, and prism size.

Varying $\theta=\theta_{A}^{f}$, we can obtain one more scenario of asymmetric beaming, in which a well pronounced transmission cannot be assigned to the piecewise-linear channel in the lower half-space for the backward-case illumination. An example is presented in Fig. 4 for $\theta=15^{\circ}$. For the forward-case illumination, we obtain the beam narrowing similar to that for $\theta=10^{\circ}$. Now we have $\delta \approx\left|\theta_{A}^{f}-\gamma_{B}^{f}\right| \approx 13^{\circ}$. Despite the allowed coupling of the incident beam to the mode(s) of $\mathrm{PhC}$ in the backward illumination case and nonvanishing reflected field on the right side from the $\mathrm{PhC}$ prism and transmitted field below its lower side, there is no signature of a distinguishable beam in the lower half-space. Possible reasons include the peculiar scattering on the prism's rods and effects of the illumination spot size. This beaming scenario is similar to some of the AT scenarios in $\mathrm{PhC}$ gratings, in which transmission is well suppressed for one of the two incidence directions, in spite of being formally allowed [2]. Although some leakage from the longer interface to the lower half-space is observed, it can be avoided by a further parameter adjustment. Note that beam behavior such as that in Figs. 2 and 4 has not been observed in the studied structure in the vicinity of $k a=4.25$ for TE polarization (not shown).

To summarize, we studied asymmetric beaming in a single piecewise-linear propagation channel enabled by the specific properties of a dielectric-rod $\mathrm{PhC}$ prism. The basic refraction 

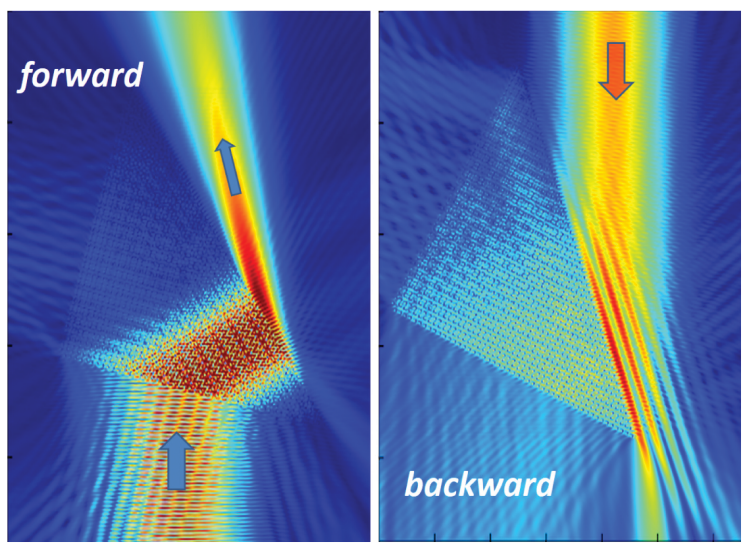

Fig. 4. Map of electric field magnitude at $k a=4.25$ and $\theta=15^{\circ}$, for forward (left panel) and backward (right panel) illumination cases; TM polarization. The prism is rotated to align in the incidence direction with the vertical direction; $\beta=45^{\circ}$.

feature contributing to the resulting hybrid refractiondiffraction mechanism is that the used FB mode shows nearly circular EFCs located around $\Gamma$ point and left-handed behavior with the equivalent index of refraction about -0.5 . The role of first-negative-order diffractions at the longer $(\Gamma-\mathrm{M})$ interface is the creation of the coupling conditions, at which desired modifications of the outgoing wave's direction and angular spectrum are possible. The wave energy can mainly be localized within one piecewise-linear path, yielding the single equivalent channel. Some other (auxiliary) channels may exist, but do not significantly affect the resulting transmission scenarios. The question whether the incident and outgoing beams can be exactly parallel in the asymmetric beaming regime within one channel (e.g., by adjusting $\theta$ and $\beta$ ) still needs an answer. The use of oblique incidence makes possible the switching between two different regimes, i.e., the one with different beam widths in the forward and backward illumination cases and the other, in which a well pronounced beaming appears only in the former case. The presented results demonstrate, among others, how close we can approach the nonreciprocal isolation regime that only needs a single linear channel. Indeed, we have the physical situation with a single piecewise-linear-not linear-channel, which is the price for staying within the linear optics approach. The obtained results promise advanced multifunctional scenarios in prism-shaped structures. Similar operating regimes in 3D photonic structures may show potential for on-chip integration, especially when directionally selective beaming are required, and will be a subject of future research.

Funding. Türkiye Bilimsel ve Teknolojik Araştirma Kurumu (2221, Tubitak Visiting Scientist Fellowship Program); Narodowe Centrum Nauki (2015/17/B/ST3/00118).

Disclosures. The authors declare no conflicts of interest.

Data Availability. Data underlying the results presented in this paper are not publicly available at this time but may be obtained from the authors upon reasonable request.

\section{REFERENCES}

1. T. Xu and H. J. Lezec, Nat. Commun. 5, 4141 (2014).

2. A. E. Serebryannikov, K. Alici, T. Magath, A. O. Cakmak, and E. Ozbay, Phys. Rev. A 86, 053835 (2012).
3. M. Stolarek, D. Yavorskiy, R. Kotyński, C. J. Z. Rodrguez, J. Łusakowski, and T. Szoplik, Opt. Lett. 38, 839 (2013).

4. B. Yao, X. Zang, Z. Li, L. Chen, J. Xie, Y. Zhu, and S. Zhuang, Photonics Res. 8, 830 (2020).

5. M. J. Lockyear, A. P. Hibbins, K. R. White, and J. R. Sambles, Phys. Rev. E 74, 056611 (2006).

6. X. Wu, G. Yuan, R. Zhu, J. Wang, F. Gao, F. Chen, and Y. Hou, Chin. Phys. Lett. 37, 044205 (2020).

7. D. L. Sounas and A. Alù, Nat. Photonics 11, 774 (2017).

8. D. Jalas, A. Petrov, M. Eich, W. Freude, S. Fan, Z. Yu, R. Baets, M. Popović, A. Melloni, J. D. Joannopoulos, M. Vanwolleghem, C. R. Doerr, and H. Renner, Nat. Photonics 7, 579 (2013).

9. J. Shi, H. Ma, C. Guan, Z. Wang, and T. Cui, Phys. Rev. B 89, 165128 (2014).

10. V. Liu, D. A. Miller, and S. Fan, Opt. Express 20, 28388 (2012).

11. A. Serebryannikov, T. Magath, and K. Schuenemann, Phys. Rev. E 74, 066607 (2006).

12. A. Mandatori, M. Bertolotti, and C. Sibilia, J. Opt. Soc. Am. B 24, 685 (2007).

13. H. Gao, G. Wei, E. Herrmann, H. Huang, Z. Zheng, and X. Wang, Opt. Express 28, 9702 (2020).

14. Ł. Zinkiewicz, J. Haberko, and P. Wasylczyk, Opt. Express 23, 4206 (2015).

15. P. Rodríguez-Ulibarri, M. Beruete, M. Navarro-Cía, and A. E. Serebryannikov, Phys. Rev. B 88, 165137 (2013).

16. A. E. Serebryannikov, H. Hajian, M. Beruete, E. Ozbay, and G. A. E. Vandenbosch, Opt. Mater. Express 8, 3887 (2018).

17. G. Kenanakis, A. Xomalis, A. Selimis, M. Vamvakaki, M. Farsari, M. Kafesaki, C. M. Soukoulis, and E. N. Economou, ACS Photonics 2, 287 (2015).

18. Y. Fu, L. Xu, Z. H. Hang, and H. Chen, Appl. Phys. Lett. 104, 193509 (2014).

19. A. E. Serebryannikov, E. Ozbay, and S. Nojima, Opt. Express 22, 3075 (2014).

20. S. Cakmakyapan, A. E. Serebryannikov, H. Caglayan, and E. Ozbay, Opt. Lett. 35, 2597 (2010).

21. M. Habib, A. E. Serebryannikov, H. Caglayan, G. A. E. Vandenbosch, and E. Ozbay, Plasmonics. 14, 721 (2019).

22. Y. Ling, L. Huang, W. Hong, T. Liu, Y. Sun, J. Luan, and G. Yuan, Opt. Express 25, 13648 (2017).

23. F. T. Gundogdu, A. E. Serebryannikov, A. O. Cakmak, and E. Ozbay, Opt. Express 23, 24120 (2015).

24. C. Lu, X. Hu, H. Yang, and Q. Gong, Opt. Lett. 36, 4668 (2011).

25. C. Lu, X. Hu, Y. Zhang, Z. Li, X. Xu, H. Yang, and Q. Gong, Appl. Phys. Lett. 99, 051107 (2011).

26. A. Song, T. Chen, X. Wang, and Y. Xi, Phys. Lett. A 381, 2283 (2017).

27. A. Song, J. Li, C. Shen, X. Peng, X. Zhu, T. Chen, and S. A. Cummer, Appl. Phys. Lett. 114, 121902 (2019).

28. A. Cicek, M. B. Yucel, O. A. Kaya, and B. Ulug, Opt. Lett. 37, 2937 (2012).

29. C. Wang, X.-L. Zhong, and Z.-Y. Li, Sci. Rep. 2, 674 (2012).

30. W. Liu, B. Wang, Y. Zhang, J. Wang, M. Zhao, F. Guan, X. Liu, L. Shi, and J. Zi, Phys. Rev. Lett. 123, 116104 (2019).

31. M. Gumus, I. H. Giden, and H. Kurt, Opt. Lett. 43, 2555 (2018).

32. V. Vashistha, M. Krawczyk, A. E. Serebryannikov, and G. A. E. Vandenbosch, Opt. Lett. 44, 4725 (2019).

33. J. Xie, S. Liang, J. Liu, P. Tang, and S. Wen, Ann. Phys. 532, 2000035 (2020).

34. W. Ji, T. Cai, G. Wang, H. Li, C. Wang, H. Hou, and C. Zhang, Opt. Express 27, 2844 (2019).

35. B. Tang, Z. Li, Z. Liu, F. Callewaert, and K. Aydin, Sci. Rep. 6, 39166 (2016).

36. Y. Ge, H.-X. Sun, S.-Q. Yuan, and Y. Lai, Appl. Phys. Lett. 112, 243502 (2018).

37. W. Liu, W. Wu, L. Huang, Y. Ling, C. Ba, S. Li, Z. Chun, and H. Li, Opt. Express 27, 33399 (2019).

38. Lumerical, www.lumerical.com/tcadproducts/fdtd/.

39. C. Ribot, M.-S. L. Lee, S. Collin, S. Bansropun, P. Plouhinec, D. Thenot, S. Cassette, B. Loiseaux, and P. Lalanne, Adv. Opt. Mater. 1, 489 (2013). 